\title{
Derechos de agua y gestión por cuencas en México. El caso del río Sonora
}

Introducción

Este artículo analiza el papel de los derechos de agua en la gestión por cuenca. Esta es considerada como un recurso de uso común donde la intervención estatal y el registro de los derechos de agua pueden ser aprovechados para poner límites a las extracciones y evitar la sobreexplotación del recurso. Para el análisis, se hace un repaso de las ideas de la gestión por cuenca y se revisa el marco legal de los derechos del agua en México; después se revisa una base de datos de derechos de agua de la cuenca del río Sonora; y al final del trabajo se presentan los hallazgos. Entre ellos se encuentran las discrepancias entre los principios del marco legal y la operación concreta de los derechos de agua, así como el desaprovechamiento de estos últimos para ejecutar la gestión por cuencas.

Palabras clave: derechos de agua, gestión por cuencas, recursos de uso común, río Sonora, México.

- Profesor-investigador Titular C de El Colegio de Sonora

npineda@colson.edu.mx

- Profesor-investigador Titular C de El Colegio de Sonora

jmoreno@colson.edu.mx

- Profesor Titular B de El Colegio de Sonora asalazar@colson.edu.mx

Asociada de investigación de la Universidad de Arizona

americalutz@email.arizona.edu denomina la "tragedia de los comunes" (Ostrom, 1990: 2); en otras palabras, en la sobreutilización de las aguas superficiales, la sobreextracción de las aguas subterráneas y el deterioro ecológico general de la cuenca, por lo cual un beneficio de corto plazo resulta en un perjuicio general a mediano o largo plazo.

En esta situación cada usuario se ubica dentro de un arreglo institucional que lo impulsa a extraer o aprovechar agua sin límites, en un entorno donde la disponibilidad de agua es limitada. El destino de este arreglo institucional es la ruina, ya que los usuarios están inmersos en una competencia, cada quien persiguiendo su propio interés dentro de una sociedad en la que el recurso es propiedad de todos (Hardin, 1968).

Para tratar de resolver el problema de la tragedia de los comunes y establecer un modelo de gestión más sustentable para recursos de 
uso común (RUC), como es el caso de las cuencas, una de las soluciones más frecuentes es recurrir a la intervención del Estado, o lo que Ostrom llama el Leviatán. Esta 'solución' consiste en el uso del poder público para fijar límites a los usuarios y de esta manera evitar la ruina de los comunes y el deterioro de la cuenca. En particular, este es el modelo de gestión que se ha adoptado en México, a fin de llevar a cabo de gestión de las cuencas hidrológicas, para promover la conservación del recurso y evitar la extracción ilimitada o descontrolada del recurso, tanto de los cuerpos superficiales de agua como de las aguas subterráneas.

El modelo de gestión mexicano se desprende del principio de propiedad originaria de la nación sobre los recursos naturales, establecido por el artículo 27 de la Constitución Mexicana de 1917. Este principio establece que la explotación, uso o aprovechamiento de los recursos y aguas nacionales requiere de una concesión expedida por el ejecutivo federal, es decir, el presidente de la república. Por "aguas nacionales" se entiende prácticamente todo cuerpo de agua relevante en el país. ${ }^{1}$ Por lo tanto, todo aprovechamiento de

\footnotetext{
I. El artículo 27 (párrafo reformado DOF 2I-04-1945, 20-0I-1960) decreta que "son propiedad de la Nación las aguas de los mares territoriales en la extensión y términos que fije el Derecho Internacional; las aguas marinas interiores; las de las lagunas y esteros que se comuniquen permanente $o$ intermitentemente con el mar; las de los lagos interiores de formación natural que estén ligados directamente a corrientes constantes; las de los ríos y sus afluentes directos o indirectos, desde el punto del cauce en que se inicien las primeras aguas permanentes, intermitentes o torrenciales, hasta su desembocadura en el mar, lagos, lagunas o esteros de propiedad nacional; las de las corrientes constantes o intermitentes y sus afluentes directos o indirectos, cuando el cauce de aquéllas en toda su extensión o en parte de ellas, sirva de límite al territorio nacional o a dos entidades federativas, o cuando pase de una entidad federativa a otra o cruce la línea divisoria de la República; la de los lagos, lagunas o esteros cuyos vasos, zonas o riberas, estén cruzadas por líneas divisorias de dos o más entidades o entre la República y un país vecino, o cuando el límite de las riberas sirva de lindero entre dos entidades federativas o a la República con un país vecino; las de los manantiales que broten en las playas, zonas marítimas, cauces, vasos o riberas de los lagos, lagunas o esteros de propiedad nacional, y las que se extraigan de las minas; y los cauces, lechos o riberas de los lagos y corrientes interiores en la extensión que fija la ley. Las aguas del subsuelo pueden ser libremente alumbradas
} 
agua debe ser autorizado por las autoridades en la materia, pues en el derecho mexicano no existe ni la propiedad privada completa del agua ni mercados de agua propiamente dichos que permitan su venta y enajenación sin la intervención del Estado.

Roemer (1997: 82) clasifica el modelo mexicano de organización de los derechos de agua como administrativo, y lo contrasta con el de cuasi mercado que existe en el estado de California (EEUU) y el de mercado que se aplica en Chile. El modelo administrativo mexicano, que más bien podemos denominar estatal, se caracteriza por la existencia de una autoridad administrativa centralizada que es la 'propietaria' de los derechos de agua y que asigna permisos de consumo y determina las condiciones de uso. Uno de los principales señalamientos que se ha hecho a este modelo es que tiende a privilegiar las preocupaciones políticas sobre las consideraciones meramente económicas; lo cual no garantiza que el agua se destine a los usos más productivos (Roemer, 1997: 16).

Con el propósito de afinar más el modelo de gestión para las cuencas hidrográficas, la Ley de Aguas Nacionales (LAN) estableció desde 1992 que "la cuenca hidrológica conjuntamente con los acuíferos, constituye la unidad de gestión de los recursos hídricos" (LAN, 1992, art. 3, fracción IV). Esta disposición fue ratificada y ampliada en las reformas de 2004, en las cuales se establece, como uno de los principios básicos que sustentan la política hídrica nacional,

mediante obras artificiales y apropiarse por el dueño del terreno, pero cuando lo exija el interés público o se afecten otros aprovechamientos; el Ejecutivo Federal podrá reglamentar su extracción y utilización y aún establecer zonas vedadas, al igual que para las demás aguas de propiedad nacional. Cualesquiera otras aguas no incluidas en la enumeración anterior, se considerarán como parte integrante de la propiedad de los terrenos por los que corran o en los que se encuentren sus depósitos, pero si se localizaren en dos o más predios, el aprovechamiento de estas aguas se considerará de utilidad pública, y quedará sujeto a las disposiciones que dicten los Estados”. 
"la gestión integrada de los recursos hídricos por cuenca hidrológica" (LAN, 2004, art. 14 Bis 5, fracc. II). Además, la LAN 2004 estipula que "compete al Ejecutivo Federal [...] Reglamentar por cuenca hidrológica y acuífero [cursivas nuestras], el control de la extracción así como la explotación, uso o aprovechamiento de las aguas nacionales del subsuelo, inclusive las que hayan sido libremente alumbradas, y las superficiales [...]". En adición, el artículo 7 de la misma declara de utilidad pública "la gestión integrada de los recursos hídricos, superficiales y del subsuelo, a partir de las cuencas" (LAN, 2004 art. 7, fracc. I). Inmediatamente después declara que también es de utilidad pública "la protección, mejoramiento, conservación y restauración de cuencas hidrológicas, acuíferos, cauces, vasos y demás depósitos de agua de propiedad nacional, zonas de captación de fuentes de abastecimiento, zonas federales, así como la infiltración natural o artificial de aguas para reabastecer mantos acuíferos" (LAN, 2004, art. 7, fracc. II).

Entonces, al menos en los textos legales, está claramente establecida la gestión por cuencas y que es el Estado, a través de las agencias y dependencias del ejecutivo, quien se encarga de tomar las medidas para controlar las extracciones y poner límite al aprovechamiento de los recursos hídricos a fin de promover la protección y conservación del recurso.

Uno de los instrumentos de la gestión del agua es la concesión de derechos y asignaciones de agua. La función de planeación y control de los derechos de agua asignada al ejecutivo mexicano, así como la administración de estos derechos que lleva a cabo la Comisión Nacional del Agua (CONAGUA), a través del Registro Público de Derechos de Agua (REPDA), implican retos y responsabilidades técnicas muy importantes para cumplir lo establecido por la ley y para lograr que el agua se aproveche de manera eficiente, evitando los derroches y desperdicios y alcanzando la sus- 
Derechos de agua y gestión por cuencas en México. El caso del río Sonora

tentabilidad con el mayor grado posible de bienestar social. El problema es que, en la práctica, aunque el propósito de estas regulaciones sea la protección y conservación de los recursos hídricos, el control de las extracciones y aprovechamientos de agua es deficiente. Panayotou (1997) expresa que "la asignación deficiente de la propiedad y del uso de las aguas nacionales, las carencias en los sistemas de administración y en el sistema tributario son las principales causas de la cobertura insuficiente de agua".

Así pues, el objetivo de este artículo es analizar el papel de los derechos de agua en la gestión por cuenca hidrográfica. Se trata principalmente de observar si la intervención estatal está ayudando a resolver el problema de la tragedia de los recursos de uso común, en el caso de las cuencas hidrográficas, y si verdaderamente el registro y control de los derechos de agua son aprovechados para poner límites a las extracciones y evitar la sobreexplotación del recurso. Para observar ese problema se estudia el caso de la cuenca de río Sonora. Las preguntas que guían el trabajo son: 1) ¿qué tanto el otorgamiento y registro de los derechos de agua ha obedecido a la orientación de gestión con base en cuencas y acuíferos? y 2) ¿han servido los derechos de agua para controlar y limitar las extracciones y aprovechamientos de agua, de modo que se alcance un mejor equilibrio en la cuenca y los acuíferos?

\section{La gestión de recursos hídricos por cuenca}

Aunque no ha sido la manera predominante de gestión, en algunos países el agua se ha manejado de manera integrada desde hace mucho tiempo. Por ejemplo, en España, en Valencia, los tribunales participativos de usuarios han funcionado por lo menos desde el siglo X (Rahaman y Varis, 2005). También de acuerdo con esos autores, España es probablemente el primer país en gestionar el agua por 
cuencas al adoptar en 1926 el sistema de confederaciones hidrográficas. Un antecedente muy mencionado es la Comisión del Valle del Tennessee, de la década de 1940, en los Estados Unidos. Sin embargo, el enfoque fue propuesto por primera vez a nivel internacional en la Conferencia Internacional sobre Agua del Mar de la Plata, organizada por las Naciones Unidas en 1977. Posteriormente este fue retomado y desarrollado en las conferencias de Dublín y de Río de Janeiro de 1992 (Rahaman y Varis, 2005).

El enfoque de la Gestión Integrada de Recursos Hídricos (GIRH) está orientado a la gestión eficiente y al desarrollo de los recursos hídricos de una manera sustentable y equilibrada, tomando en cuenta tanto los intereses sociales y económicos de los usuarios como la demanda de agua del medio ambiente. Este enfoque enfatiza la participación de los diversos sectores en los procesos de decisión y formulación de políticas, con el fin de establecer una buena gobernanza y de crear un marco legal e institucional efectivo como la vía para decisiones más equitativas y sustentables (Global Water Partnership 2009: 10). La GIRH es una estrategia para el desarrollo y la gestión del agua que hace un balance de las necesidades económicas y sociales, al tiempo que asegura la protección de ecosistemas para generaciones futuras. La GIRH es también definida como "un proceso que promueve la gestión y desarrollo coordinado del agua, la tierra y los recursos relacionados, con el fin de maximizar el bienestar social y económico resultante de manera equitativa, sin comprometer la sostenibilidad de los ecosistemas" (Global Water Partnership 2009: 18). De acuerdo con el planteamiento de Dourejeanni (2004), la GIRH es un componente de la gestión de los recursos naturales y esta, a su vez, es parte de la gestión ambiental. El control y administración de los derechos de agua puede ser una herramienta muy valiosa y definitiva para la gestión por cuencas. Por otra 
Derechos de agua y gestión por cuencas en México. El caso del río Sonora

parte, la GIRH tiene como sus componentes la gestión del agua por sectores.

En México, aunque existen experiencias anteriores (Barkin y King, 1975), la gestión por cuencas se adoptó oficialmente en la LAN de 1992. De acuerdo con esta (LAN, 2004, art. 3, fracc. XVI), por cuenca hidrográfica se entiende:

la unidad del territorio, diferenciada de otras unidades, normalmente delimitada por un parte aguas o divisoria de las aguas -aquella línea poligonal formada por los puntos de mayor elevación en dicha unidad-, en donde ocurre el agua en distintas formas, y ésta se almacena o fluye hasta un punto de salida que puede ser el mar u otro cuerpo receptor interior, a través de una red hidrográfica de cauces que convergen en uno principal, o bien el territorio en donde las aguas forman una unidad autónoma o diferenciada de otras, aun sin que desemboquen en el mar. En dicho espacio delimitado por una diversidad topográfica, coexisten los recursos agua, suelo, flora, fauna, otros recursos naturales relacionados con éstos y el medio ambiente.

La cuenca hidrológica a su vez está integrada por subcuencas y estas últimas están integradas por microcuencas. Hay que agregar que estrechamente vinculado con el concepto de cuenca, la misma LAN (2004, art. 3, fracc. XVI) define además el concepto región hidrológica como:

[el] área territorial conformada en función de sus características morfológicas, orográficas e hidrológicas, en la cual se considera a la cuenca hidrológica como la unidad básica para la gestión de los recursos hídricos, cuya finalidad es el agrupamiento y sistematización de la información, análisis, diagnósticos, programas y acciones en relación con la ocurrencia del agua en cantidad y calidad, así como su explotación, uso o aprovechamiento.

De este modo, para fines administrativos y de sistematización de la información, la cuenca es sustituida por la región 
hidrológica que puede estar integrada por una o varias cuencas hidrológicas y cuyos límites normalmente son distintos de las divisiones políticas estatales o municipales. Una o varias regiones hidrológicas integran una región hidrológica-administrativa. Ese mismo documento define la gestión del agua como:

Proceso sustentado en el conjunto de principios, políticas, actos, recursos, instrumentos, normas formales y no formales, bienes, derechos, atribuciones y responsabilidades, mediante el cual coordinadamente el Estado, los usuarios del agua y las organizaciones de la sociedad, promueven e instrumentan para lograr el desarrollo sustentable en beneficio de los seres humanos y su medio social, económico y ambiental, (I) el control y manejo del agua y las cuencas hidrológicas, incluyendo los acuíferos, por ende su distribución y administración, (2) la regulación de la explotación, uso o aprovechamiento del agua, y (3) la preservación y sustentabilidad de los recursos hídricos en cantidad y calidad, considerando los riesgos ante la ocurrencia de fenómenos hidrometeorológicos extraordinarios y daños a ecosistemas vitales y al medio ambiente. La gestión del agua comprende en su totalidad a la administración gubernamental del agua (LAN, 2004, art. 3, fracc. XXVIII).

En el presente trabajo consideramos que los derechos de agua pueden y deben jugar un papel central dentro del enfoque de GIRH por cuencas. Este papel o función se puede definir por medio de dos principios. El primero de ellos es que la cuenca es el espacio básico y primordial de la planeación y gestión de los recursos hídricos. Con base en este principio los derechos deben estar adscritos y definidos en función de una cuenca. En la contraparte de este principio, con frecuencia el registro y asignación de derechos de agua responde a otros criterios, como la división política o los sectores económicos, lo cual no permite que se haga un inventario de consumos y uso del agua con base en la cuenca. El segundo es que la información es una condición 
Derechos de agua y gestión por cuencas en México. El caso del río Sonora

y herramienta de la planeación y la gestión por cuenca. Esto implica, entonces, que la gestión por cuencas requiere de un sistema de información sobre el ciclo del agua que sirva de insumo a la planeación y gestión de los derechos y asignaciones del agua. En este sistema de información los derechos de agua permiten cuantificar las extracciones e intervenciones sociales en el ciclo del agua y posibilitan la realización de balances y diagnósticos de los consumos y asignaciones de agua. Sin esta información no es posible la planeación ni la gestión de la cuenca (Global Water Partnership, 2009: 83-92).

\section{Los derechos de agua en México}

En este estudio se entiende por "derecho de agua" la autorización otorgada por la autoridad legal correspondiente para acceder a la explotación, uso o aprovechamiento del recurso hídrico de propiedad nacional, por medio de un título o documento oficial que ha sido registrado en el REPDA. En este sentido, tanto la "asignación" como la "concesión" de derechos de agua se refieren a los títulos o documentos que avalan esta autorización. La diferencia entre asignación y concesión estriba en que la primera se otorga a los municipios, a los estados o al Distrito Federal y está destinada a los servicios de agua con carácter público, urbano o doméstico (LAN, art. 3, VIII), mientras que la concesión se otorga a las personas físicas o morales de carácter público y privado (LAN, art. 3, XIII). Otra diferencia es que las asignaciones no pueden ser objeto de transmisión (LAN, art. 20), mientras que las concesiones pueden ser transferidas entre personas físicas o morales, bien por la vía sucesoria o por adjudicación judicial (Reglamento de la Ley Aguas Nacionales, RLAN, arts. 64 y 71; DOF, 12 de enero de 1994). La transmisión de aguas superficiales solo puede hacerse dentro de la misma cuenca y la de aguas subterráneas solo dentro del mismo 
acuífero (RLAN, art. 64). En el resto de este trabajo, al hablar de concesión, se estarán incluyendo tanto las asignaciones como las concesiones.

Este régimen de intervención estatal en el acceso al agua ha implicado la planeación de la gestión del recurso realizada por el gobierno federal, así como el establecimiento de registros y controles de las concesiones. Asimismo, a partir del hecho de que el Ejecutivo Federal expide los títulos, se infiere que este tiene la responsabilidad de velar por el interés general de la nación y, por lo tanto, de prever y planear el destino más apropiado para los usos del agua.

La ley establece que el criterio para otorgar las concesiones es la disponibilidad media anual del agua en el lugar en que se solicita. Este criterio pudiera significar un problema en tiempos de sequía cuando la disponibilidad del recurso está por debajo de la media y el conjunto de los derechos asignados significa un consumo agregado superior al disponible. Asimismo, conforme a la planeación y la normatividad existente, la disponibilidad del recurso debe revisarse cada tres años (LAN art. 22, párrafo segundo). Con la finalidad de anticiparse a posibles bajas en la disponibilidad, la LAN prevé que las concesiones expedidas señalen expresamente las condiciones de variabilidad de la fuente donde se realiza la extracción, así como sequías y otros fenómenos. Por ello, los títulos de concesión no garantizan la existencia o invariabilidad de los volúmenes que amparan (art. 22, párrafo quinto); en otras palabras, el hecho de contar con una concesión no garantiza necesariamente el acceso al recurso, porque este queda sujeto a que el recurso hídrico se encuentre disponible en la fuente o lugar de extracción.

La LAN es reiterativa en que los concesionarios deben limitar sus extracciones a la disponibilidad del recurso. Así, establece que "en ningún caso podrá el titular de una concesión o asignación disponer del agua en volúmenes mayores que los autorizados por la Autoridad del Agua"; 
Derechos de agua y gestión por cuencas en México. El caso del río Sonora

además para aumentar o modificar la extracción, el concesionario debe tramitar la expedición de un nuevo título (art. 23, párrafo tercero). También el concesionario debe avisar a la autoridad hidráulica cuando cambie o modifique el tipo de uso que le da al recurso y deberá solicitar permiso para realizar las obras que se requieren para ese nuevo tipo de aprovechamiento (LAN, art. 25, párrafo octavo).

Para reforzar la limitación en las extracciones del agua a los volúmenes concesionados, la LAN establece que los concesionarios deben contar con medidores o dispositivos para la medición directa o indirecta de las extracciones (art. 29 , fracc. II). También los concesionarios deben pagar los derechos fiscales que les correspondan de acuerdo con las extracciones, consumo y descargas volumétricas que realicen. El incumplimiento de estos pagos puede ser motivo de suspensión o revocación de la concesión (LAN, art. 29, fracc. IV). Los concesionarios deben proporcionar la información y documentación que les soliciten las autoridades del agua y deben procurar el uso eficiente y el reúso de acuerdo con las Normas Oficiales Mexicanas (LAN, art. 29, fracc. IX y $\mathrm{X})$. Por último, es importante destacar que las concesiones son temporales y pueden tener una duración de entre cinco y veinte años (LAN, art. 24). Por lo tanto, las concesiones deben ser renovadas al final del periodo de concesión y en cada caso la autoridad hidráulica tiene la posibilidad de suspenderlas o revocarlas.

\section{El Registro Público de Derechos de Agua}

La LAN del 1 de diciembre 1992 estableció en su artículo 30 el Registro Público de Derechos de Agua (REPDA). La página Web de la CONAGUA (www.conagua.gob.mx) y la Memoria Documental del REPDA le asignan al registro el objetivo o misión de "proporcionar seguridad jurídica a los concesionarios de aguas nacionales y sus bienes públicos 
inherentes, haciendo constar la titularidad de los derechos inscritos y los cambios en sus características y resolver consultas en forma eficaz, sobre los derechos de los usuarios en materia de aguas nacionales" (Comisión Nacional del Agua, s.f.).

$\mathrm{Al}$ analizar este objetivo desde el punto de vista de la GIRH, se observa que el REPDA tiene más bien la función de prevenir conflictos y discrepancias entre los usuarios y no tanto la de planear y controlar los usos y extracciones siguiendo la disponibilidad. De este modo, la seguridad jurídica que se menciona puede ser efectiva frente a otros demandantes, pero no cuando se trata de limitaciones en la disponibilidad natural del recurso. Dicho de otro modo, la garantía o certeza jurídica no opera cuando se agota el pozo o cuando se seca el río. Se infiere entonces que, de acuerdo con el marco normativo, ni los derechos ni su registro tienen la función de controlar los usos y extracciones del recurso en la búsqueda de su conservación y equilibrio.

Aunado a lo anterior, la ley tampoco establece ninguna disposición para que los usuarios procuren ahorrar o conservar las fuentes de suministro tratando de utilizar volúmenes menores a los que les fueron concesionados. Aun cuando la LAN sugiere que la concesión no se perderá si el usuario no usa la totalidad del agua concesionada, mientras las causas de esto no sean imputables al usuario (entre los concesionarios es frecuente la idea de que pueden perder la concesión si no emplean en su totalidad el recurso). ${ }^{2}$ Lo más cercano que llega a la idea de conservación del recurso es la disposición del artículo 29, fracción X, de "cumplir con los requisitos de uso eficiente del agua y realizar su reúso". Esta ausencia de política de conservación propicia que, en

2. El art. 26, fracc. III, de la LAN de 1992 y el art. 29 bis 2 , fracción $V$ de la LAN reformada de 2004 establecen que las concesiones o asignaciones se suspenden cuando el titular "no cumpla con las condiciones o especificaciones del título de concesión o asignación". 
Derechos de agua y gestión por cuencas en México. El caso del río Sonora

la práctica, las concesiones y la certeza jurídica que otorgan los derechos tiendan a ser un incentivo para utilizar o extraer en su totalidad los volúmenes de agua concesionados. Esto refuerza la noción de que el propósito o función de los derechos de agua no es el control de las extracciones ni alcanzar un equilibrio con respecto a la disponibilidad física del recurso.

En lo que se refiere a la gestión por cuencas, ni la LAN ni su reglamento establecen que los títulos de derechos de agua deban adscribirse a una cuenca. Los títulos especifican, entre otros, los datos del titular, el volumen concesionado y la fecha de registro, pero no la cuenca a la que pertenecen. Solo en el caso de aguas subterráneas se hace mención al acuífero del que se hace la extracción. Sin embargo, al referirse a la transmisión de títulos de concesiones y asignaciones, el artículo 64 del Reglamento establece que únicamente "se podrán transmitir los derechos [...] dentro de una misma cuenca, o de aguas del subsuelo dentro de un mismo acuífero". De esto se deduce que debe conocerse y definirse no solo la división política en la que se ubican los derechos, sino también, y de manera primordial, la cuenca o el acuífero al que pertenecen.

En lo que respecta a su organización administrativa, el REPDA ha oscilado entre la centralización y la descentralización. El Reglamento de la LAN del 12 de enero de 1994, en su artículo 54, disponía que se establecieran oficinas en todas las entidades federativas. Sin embargo, un oficio del Director General de la CONAGUA del 15 de junio de 1995 dispuso que con el objeto de dar mayor seguridad y calidad la fe pública registral se concentraba en la gerencia nacional del registro. Posteriormente, el 2 de noviembre de 2000 se publicó el acuerdo que delega nuevamente las funciones registrales en los gerentes regionales de la CONAGUA. En los hechos, sin embargo, toda la información y actividades sustantivas son concentradas en la gerencia nacional; las 
gerencias regionales funcionan como meras ventanillas de trámite.

La evolución del número de registros y movimientos de títulos se ha ido incrementando con el paso de los años. En una primera etapa la actividad se concentró en el registro de derechos por primera vez. Pero en una segunda etapa la actividad registral ha disminuido y se ha incrementado el registro de movimientos y transmisiones de derechos. Es así como en 1994 hubo quince mil setecientos ochenta y nueve primeros registros y solo ciento cincuenta y seis movimientos. El periodo de mayor actividad registral fue de 1995 a 1999, cuando hubo trescientos treinta mil doscientos quince registros y solo dos mil ciento treinta y cuatro movimientos. A partir del año 2000 la actividad de registros primerizos a nivel nacional comenzó a bajar, aunque empezaron a repuntar los movimientos. En el año 2002 los registros fueron únicamente veinticinco mil ochocientos ochenta y tres y los movimientos dieciocho mil quinientos cuarenta y tres. Por último, en el periodo de 2009 a 2011 los registros fueron veinticinco mil doscientos cincuenta y uno y los movimientos, setenta y un mil setecientos treinta y seis (ver Figura 1).

Al 31 de marzo de 2012, el REPDA tenía registrados un total de cuatrocientos cincuenta y siete mil ochocientos tres títulos de concesión en todo el país. De estos, veintiséis mil seiscientos ochenta y cinco (el 6\%) corresponden al Organismo de Cuenca Noroeste (OCNO), en cuya jurisdicción se ubica la cuenca del río Sonora.

La cuenca del Río Sonora, la región hidrológica y la división municipal

Cuando se trata de vincular la cuenca de un río con los derechos de agua otorgados, lo que se encuentra es que la información de los derechos no está disponible por cuenca, 
Derechos de agua y gestión por cuencas en México. El caso del río Sonora

Figura 1. Evolución de los registros y movimientos de derechos de agua

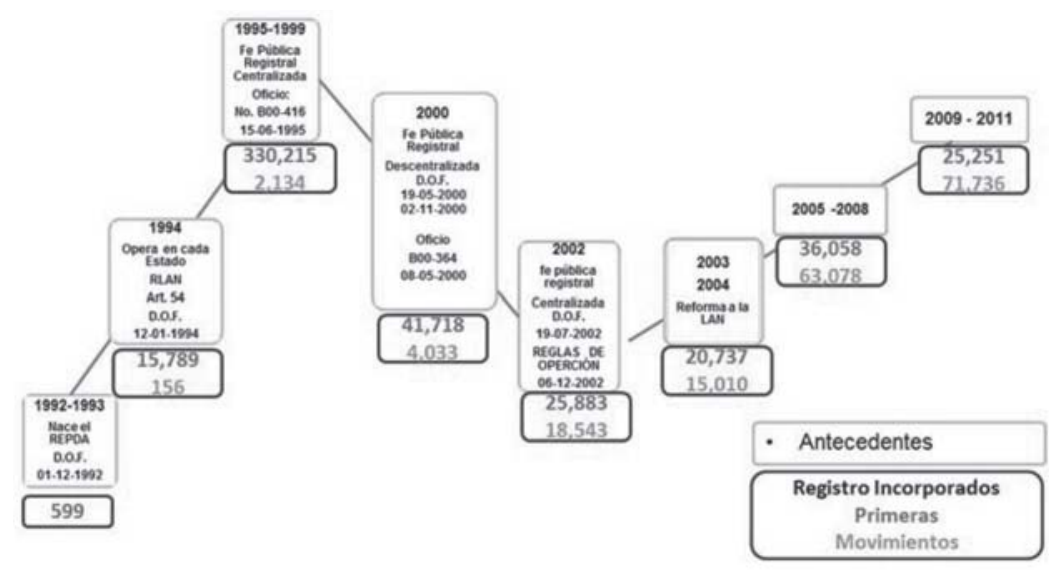

Fuente: Comisión Nacional del Agua: www.cna.gob.mx

sino solamente por región hidrológica, acuífero homologado o por división política. Los formularios de búsqueda del REPDA contienen entradas por los rubros estado, municipio, organismo de cuenca, región hidrológica, acuífero homologado, titular, título, fecha de inicio y fecha final, pero no hay una vía de acceso por el rubro cuenca.

Hay que recordar que la región hidrológica establecida por la LAN, como la base para la gestión por cuencas y para la sistematización de la información, puede comprender varias cuencas. De este modo, el río Sonora pertenece a la región hidrológica denominada Sonora Sur, que comprende las cuencas de los ríos Sonora y Yaqui.

De este modo, la información de derechos de agua que ofrece el REPDA sobre el río Sonora está agrupada con las cuencas de los ríos Mátape, Yaqui, arroyo Cocoraque y Mayo. Esta información no distingue cuáles derechos corresponden a la cuenca del río Sonora y cuáles a las otras 
Figura 2. Región Hidrológica 9, Sonora Sur

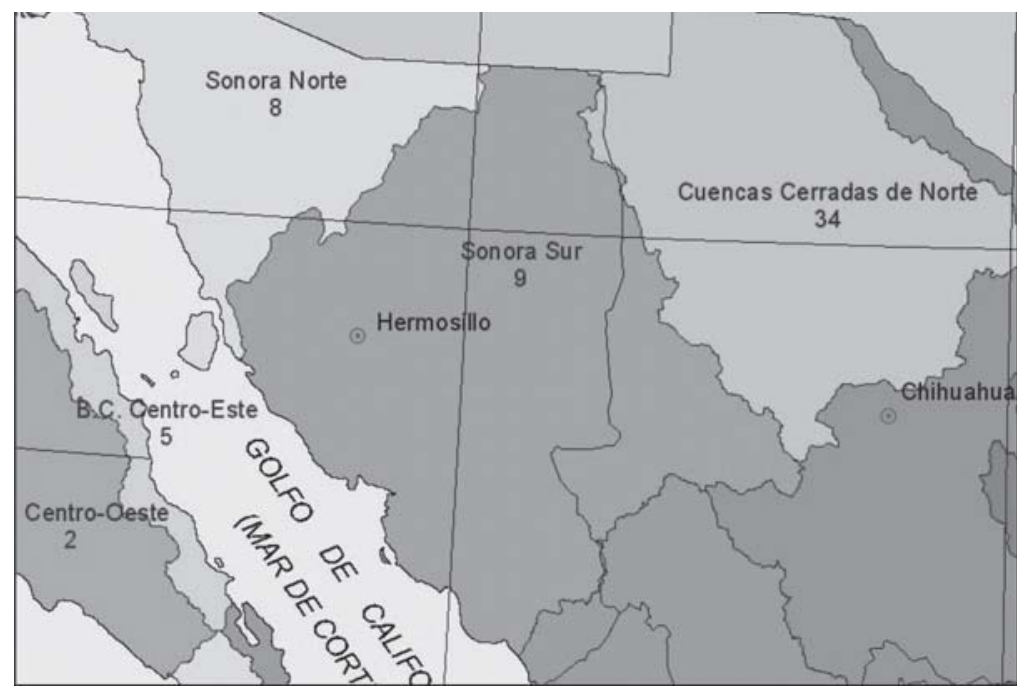

Fuente: http://siga.cna.gob.mx/mapoteca/regiones\%20hidrologicas/ mapareghidro.htm

cuencas. Si se consulta la información sobre cada título de derecho de agua tampoco se obtiene ahí la información de la cuenca a la que corresponde. Se requiere ir a los anexos del título para encontrar que un derecho particular corresponde a cierto número de cuenca que no es identificada por nombre. Por ejemplo, uno de los primeros títulos que aparece al consultar los derechos correspondientes a la región hidrológica Sonora Sur es el del titular Organismo Operador Municipal de Agua Potable, Alcantarillado y Saneamiento de Aconchi, título 02SON121876/09HMGR00, de uso público urbano, registrado el 11 de agosto de 2000. Solo al ingresar a la información específica sobre este título, se obtiene la información sobre el volumen de agua concesionado (200 592 $\mathrm{m}^{3}$ /año) y que la concesión se refiere a agua subterránea, pero no se informa la ubicación precisa del derecho. Hasta que se ingresa a los anexos de dicho título publicados en 
Derechos de agua y gestión por cuencas en México. El caso del río Sonora

Internet se obtiene la información de que la extracción a la que se refiere el título se ubica en el estado (26) de Sonora, en el municipio (1) de Aconchi, en la región hidrológica (9), Sonora Sur, en la cuenca 4, acuífero homologado (2624), Río Sonora, que corresponde a la latitud $29^{\circ} 49^{\prime} 10.00^{\prime \prime}$ y a la longitud $-110^{\circ} 14^{\prime} 32.00$ ”. En resumen, la información de la cuenca a la que pertenecen los derechos no está fácilmente accesible y la compilación manual de dicha información accediendo a los anexos de cada derecho uno por uno necesitaría mucho tiempo.

Otra vía de llegar a los derechos de agua de la cuenca del río Sonora es a través de la división municipal en la cuenca. De acuerdo con un estudio de la Comisión Estatal del Agua de Sonora (CEA Sonora), la cuenca del río Sonora se ubica enteramente dentro del estado de Sonora y comprende un total de veintisiete municipios. De estos, solo tres municipios están ubicados en su totalidad dentro de la cuenca; otros diez tienen la mayor parte de su territorio ubicada en la cuenca, mientras que otros catorce tienen solo una parte minoritaria de su territorio dentro de la cuenca (Comisión Estatal del Agua y Torynor, 2009).

Con el fin de identificar los derechos de la cuenca del río Sonora, se seleccionaron los quince municipios indicados por la Comisión Estatal del Agua en Sonora (2008), y cuyo territorio se ubica mayoritariamente dentro de la cuenca del río Sonora: Aconchi, Arizpe, Bacoachi, Banámichi, Baviácora, Cananea, Carbó, Cucurpe, Hermosillo, Huépac, Opodepe, Rayón, San Felipe de Jesús, San Miguel de Horcasitas y Ures.

A través de la consulta a la página web del REPDA entre noviembre de 2011 y enero de 2012 se construyó una base de datos de los derechos concesionados en los 15 municipios de la cuenca del río Sonora. La base contiene un total de cuatro mil novecientos sesenta y cinco registros de títulos de concesión. Al revisar dicha base, se observa que veintiséis 
Figura 3. Cuenca del río Sonora y división municipal

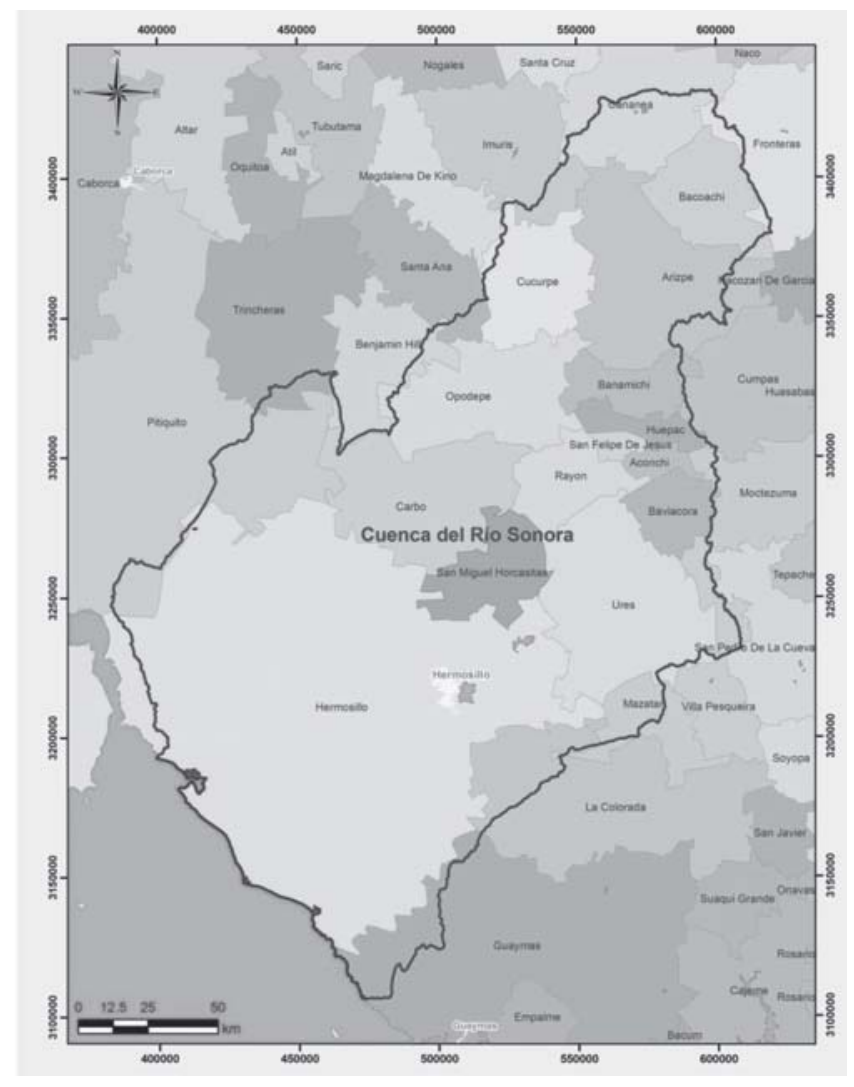

Fuente: Lucas Oroz, Comisión Nacional del Agua. Presentación sobre río Sonora, 29 de abril de 2010.

Nota: En esta figura, en el territorio de Hermosillo, el área de la cuenca incluye al río Bachoachi.

registros aparecen en blanco, es decir, no contienen ningún dato referente al volumen de agua concesionado, ni superficial ni subterráneo, y solo contienen el nombre del titular y la fecha de la concesión. Según la consulta realizada, estos registros en blanco corresponden a derechos obsoletos que no fueron renovados y ya no están vigentes. Restados los registros en blanco, la base de datos con información 
numérica consta de cuatro mil novecientos treinta y nueve registros, de los cuales cuatro mil cuatrocientos trece corresponden a concesiones de extracción de agua (superficial o subterránea), mientras que el resto se refieren a permisos de descarga de aguas residuales y zonas federales.

La información de cada título incluye: 1) municipio donde se encuentra registrado el título, 2) nombre del titular, 3) la clave de registro del título, 4) tipo de uso, 5) fecha de registro, 6) el total del volumen de agua que se concesiona en metros cúbicos por año (este volumen se desglosa además en el número de aprovechamientos superficiales y subterráneos, así como el volumen superficial y subterráneo concesionado), 7) número de puntos de descarga de aguas residuales y volumen de descarga permitido en metros cúbicos por día, y 8) número de zonas federales y superficie de zona federal en metros cuadrados involucradas en el derecho de concesión, en su caso.

Distribución espacial y temporal de los derechos de agua

Para su estudio y análisis, con base en la información municipal, los títulos se dividieron en cinco grupos correspondientes a cinco subcuencas o regiones geográficas que son: la cuenca alta, la cuenca media, el afluente San Miguel, el afluente el Zanjón y la cuenca baja.

En cuanto a la temporalidad de los registros, la base de datos comprende el periodo de 1994 a 2011. El primer registro fue realizado el 3 de mayo de 1994 y corresponde a un derecho registrado en el municipio de San Miguel de Horcasitas. En el otro extremo, el último registro fue hecho el 4 de abril de 2011 en el municipio de Hermosillo. La distribución de los títulos por año de asignación muestra que las cantidades más grandes de otorgamiento de títulos se dieron en los años de 1996 a 2000, mientras que a partir de 2001 el otorgamiento de títulos descendió considerablemente. 
Nicolás Pineda, José Luis Moreno, Alejandro Salazar y América Lutz Ley

Tabla 1. Títulos de concesión de agua, descargas de aguas residuales y zonas federales por subcuencas y municipios

\begin{tabular}{|c|c|c|c|}
\hline Subcuenca & Municipios & Títulos & Títulos \\
\hline Cuenca Alta & Cananea & 125 & 125 \\
\hline \multirow[t]{9}{*}{ Cuenca Media } & & & 1904 \\
\hline & Aconchi & 92 & \\
\hline & Arizpe & 352 & \\
\hline & Bacoachi & 202 & \\
\hline & Banamichi & 84 & \\
\hline & Baviacora & 179 & \\
\hline & Huépac & 142 & \\
\hline & $\begin{array}{l}\text { San Felipe De } \\
\text { Jesús }\end{array}$ & 26 & \\
\hline & Ures & 827 & \\
\hline \multirow[t]{6}{*}{ San Miguel } & & & 1149 \\
\hline & Cucurpe & 278 & \\
\hline & Opodepe & 316 & \\
\hline & Rayón & 216 & \\
\hline & San Miguel De & 339 & \\
\hline & Horcasitas & & \\
\hline \multirow[t]{2}{*}{ Zanjón } & & & 213 \\
\hline & Carbó & 213 & \\
\hline \multirow[t]{2}{*}{ Cuenca Baja } & & & 1548 \\
\hline & Hermosillo & 1548 & \\
\hline Totales & & 4939 & 4939 \\
\hline
\end{tabular}

Fuente: Elaboración propia con información de una base de datos de derechos agua del río Sonora de 2012 proporcionada por el REPDA

Un funcionario del Organismo de Cuenca del Noroeste en entrevista informó que el proceso de registro de los derechos de agua a partir de 1994 consistió -más bien- en el reconocimiento de los usuarios del recurso ya existentes y no fue, por lo tanto, un proceso de decisión con base en consideraciones de disponibilidad o conservación del recurso. Incluso el proceso fue lento, ya que tuvo que vencer muchas resistencias de los usuarios a proporcionar la información y a realizar los pagos correspondientes (Oroz, 2012a). De 
Figura 4. Otorgamiento de títulos de concesión en la cuenca del río Sonora

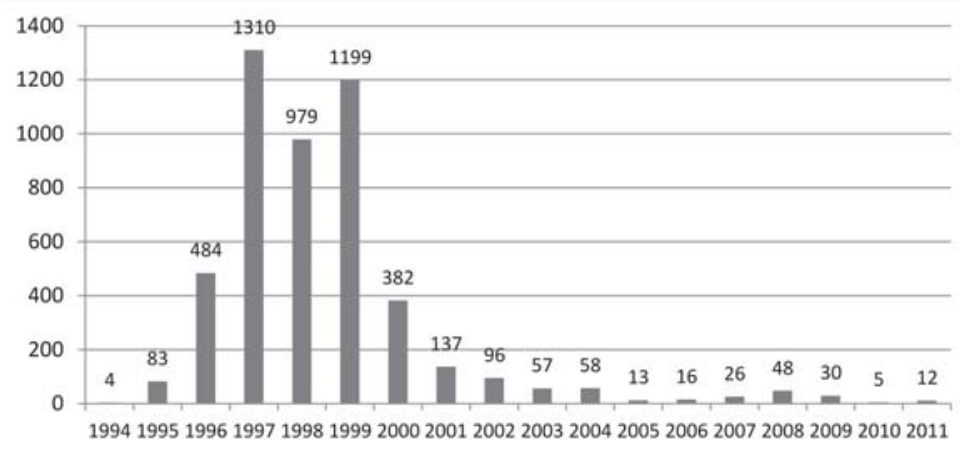

Fuente: Elaboración propia con información de una base de datos de derechos agua del río Sonora de 2012 proporcionada por el REPDA

este modo, el otorgamiento de los títulos no fue un proceso que tuviera el control sobre el número de concesiones y donde hubiera solicitudes que pudieran no ser aceptadas. Más bien se trató de un proceso en el que la CONAGUA y el REPDA hicieron campaña de convencimiento a los usuarios para que registraran sus usos y aprovechamientos.

\section{Volúmenes y usos de agua concesionados}

De acuerdo al ejercicio realizado, en los 15 municipios el total de volumen concesionado es de 1026 millones de metros cúbicos $\left(\mathrm{Mm}^{3}\right)$. De este total, la cuenca alta tiene el 3\%, la cuenca media, el 10\%, el río San Miguel, el 8\%, el río Zanjón, el 3\%, y la cuenca baja, el 76\%; o sea, la cuenca baja concentra poco más de tres cuartos del agua concesionada en esta región. Del total de volumen concesionado, el 59\% corresponde a aguas superficiales, mientras el restante $41 \%$ corresponde a aguas subterráneas. De este modo, por sí sola, la cuenca baja concentra el 92\% del agua superficial y el 53\% del agua subterránea concesionada. 
Existen dos consideraciones importantes con respecto a estas cifras. Por un lado, en la base de datos del REPDA no se localizaron las concesiones correspondientes al Distrito de Riego 051-Costa de Hermosillo (DDR 051), conocido por su importancia agrícola y por los volúmenes de agua que utiliza según estimaciones recientes (Olavarrieta et ál., 2010). Asimismo, se encontró un título de concesión a nombre del Gobierno del Estado de Sonora por $540 \mathrm{Mm}^{3}$ de agua superficial para uso público urbano; mismo que se asume es el correspondiente a la presa Abelardo L. Rodríguez en la cuenca baja (el municipio de Hermosillo), pero que dejó de funcionar debido a las casi inexistentes recargas de agua. En comunicación personal con un funcionario del Organismo de Cuenca Noroeste (Oroz, 2012b), este indicó que ya se había solicitado la extinción del título, pero aún no había sido dado de baja en el REPDA. En este análisis se utilizan los volúmenes y concesiones tal y como se obtuvieron del REPDA, y es por ello que en la cuenca baja el volumen de agua superficial y el volumen total para uso urbano pueden estar sobreestimados, mientras que el volumen para uso agrícola en esta misma sección de la cuenca puede estar subestimado.

Tabla 2. Volumen de agua concesionado por subcuenca ( $\left.m^{3} / a \tilde{n} o\right)$

\begin{tabular}{lrrrr} 
Subcuencas & \multicolumn{1}{c}{$\begin{array}{c}\text { Vol. } \\
\text { superficial }\end{array}$} & \multicolumn{1}{c}{$\begin{array}{c}\text { Vol. } \\
\text { subterráneo }\end{array}$} & $\begin{array}{c}\text { Total vol. } \\
\text { concesionado }\end{array}$ & Porcentaje \\
Cuenca Alta & 75063 & 35513115 & 35588178 & $3 \%$ \\
Cuenca Media & 35526962 & 64417428 & 99631890 & $10 \%$ \\
San Miguel & 13860399 & 71737541 & 85597939 & $8 \%$ \\
Zanjón & 25550 & 30012820 & 30250719 & $3 \%$ \\
Cuenca Baja & 553683673 & 224803327 & 775496627 & $76 \%$ \\
Total general & 603171646 & 426484231 & 1026565353 & $100 \%$ \\
Porcentaje & $59 \%$ & $41 \%$ & $100 \%$ & \\
\hline
\end{tabular}

Fuente: Elaboración propia con información de una base de datos de derechos agua del río Sonora de 2012 proporcionada por el REPDA 
Si rehiciéramos el análisis de los datos omitiendo el título de concesión de $540 \mathrm{Mm}^{3}$ de aguas superficiales para uso público urbano cuya baja se encuentra en proceso, e incluyendo el volumen concesionado al DDR 051-Costa de Hermosillo por aproximadamente $325 \mathrm{Mm}^{3}$ de agua subterránea para uso agrícola (CONAGUA y OCNO, 2007), entonces:

- El volumen de agua superficial concesionada en la cuenca baja sería de 13.7 millones de $\mathrm{m}^{3}$; y no de 553.7 millones de $\mathrm{m}^{3}$, como se obtuvo del análisis de los datos del REPDA resumidos en Tabla 2.

- El volumen de agua subterránea concesionada en la cuenca baja sería de casi $550 \mathrm{Mm}^{3}$; es decir, poco más del doble del volumen que se obtuvo del análisis de los datos.

- El volumen total concesionado para la cuenca baja sería entonces de poco más de $560 \mathrm{Mm}^{3}$, y no de 775.5 como se obtuvo en Tabla 2.

- Finalmente, el volumen total concesionado en toda la cuenca del río Sonora disminuiría de $1026.5 \mathrm{Mm}^{3}$ a casi $812 \mathrm{Mm}^{3}$.

En lo que se refiere a los usos, la LAN define cinco tipos: el agrícola, el doméstico, el industrial, el pecuario y el público urbano. Sin embargo, en la base de datos también aparecen los tipos: múltiple, agroindustrial, y de servicios, pero sin definir. A partir de la observación de estos títulos se deduce que los derechos múltiples corresponden a combinaciones de dos o más de los cinco usos especificados en la ley; por ejemplo, concesiones para uso doméstico y agrícola, doméstico y pecuario, pecuario y agrícola, entre otras. De la misma manera, el uso agroindustrial corresponde a la combinación del uso agrícola y el industrial. Por último el de servicios se refiere al autoabastecimiento de agua para edificaciones de comercios o servicios (públicos o privados), que no son industriales y que se encuentran separados de los centros urbanos. En este caso están aeropuertos, moteles o almacenes ubicados fuera de la 
ciudad, entre otros establecimientos. Otro uso que aparece en la base de datos es el de acuacultura. Aunque en este caso no se trata propiamente de una concesión de uso de agua sino de una autorización para descargar aguas residuales derivadas de esta actividad.

Tabla 3. Tipos de usos contemplados en la Ley de Aguas Nacionales

Tipo de uso Descripción

Agrícola La aplicación de agua nacional para el riego destinado a la producción agrícola, y la preparación de esta para la primera enajenación, siempre que los productos no hayan sido objeto de transformación industrial.

Doméstico La aplicación de agua nacional para el uso particular de las personas y del hogar, riego de sus jardines y de árboles de ornato, incluyendo el abrevadero de animales domésticos, siempre que esto no constituya una actividad lucrativa, en términos del artículo 115 de la Constitución Política de los Estados Unidos Mexicanos.

Industrial La aplicación de aguas nacionales en fábricas o empresas que realicen la extracción, conservación o transformación de materias primas o minerales, el acabado de productos o la elaboración de satisfactores, así como el agua que se utiliza en parques industriales, calderas, dispositivos para enfriamiento, lavado, baños y otros servicios dentro de la empresa, las salmueras que se utilizan para la extracción de cualquier tipo de sustancias y el agua aun en estado de vapor, que sea usada para la generación de energía eléctrica o para cualquier otro uso o aprovechamiento de transformación.

Pecuario La aplicación de aguas nacionales para la cría y engorda de ganado, aves de corral y otros animales, y su preparación para la primera enajenación, siempre que no comprendan la transformación industrial; no incluye el riego de pastizales.

Público La aplicación de agua nacional para centros de población y urbano asentamientos humanos, a través de la red municipal.

Fuente: Ley de Aguas Nacionales, Título Primero, Fracciones LIII, LVI, LVIII, LIX y LX. DOF, 29 de abril de 2004. 
De este modo, tenemos que el principal uso de la cuenca es el uso público urbano que concentra el $60 \%$ del volumen concesionado. Le sigue el uso agrícola, con el 31\% del agua. Estos dos usos concentran el 91\% del agua concesionada, mientras que los otros usos tienen solo el 9\% del agua, según se observa en Tabla 4.

Tabla 4. Usos del agua en el río Sonora $\left(\mathrm{Mm}^{3} /\right.$ año)

\begin{tabular}{lrrrrrrr} 
Usos & Cuenca & Cuenca & San & Zanjón & Cuenca & Total por & $\%$ \\
& Alta & Media & Miguel & & Baja & \multicolumn{1}{c}{ uso } & \\
Agrícola & 1.410 & 90.595 & 69.324 & 27.003 & 132.825 & 321.158 & 31 \\
Agroindustrial & - & - & - & - & 0.050 & 0.050 & 0 \\
Doméstico & 0.001 & 0.028 & 0.017 & - & 0.054 & 0.100 & 0 \\
Industrial & 28.156 & 0.002 & 0.180 & - & 4.608 & 32.947 & 3 \\
Múltiple & 3.790 & 4.674 & 14.047 & 2.630 & 26.540 & 51.682 & 5 \\
Pecuario & 0.352 & 1.223 & 1.003 & 0.307 & 2.412 & 5.297 & 1 \\
Público urbano & 1.879 & 3.068 & 1.016 & 0.311 & 607.730 & 614.003 & 60 \\
Servicios & - & 0.041 & 0.010 & - & 1.278 & 1.329 & 0 \\
Total & 35.588 & 99.632 & 85.598 & 30.251 & 775.497 & 1026.565 & 100 \\
\hline
\end{tabular}

Fuente: Elaboración propia con información de una base de datos de derechos agua del río Sonora de 2012 proporcionada por el REPDA

Si rehiciéramos el análisis omitiendo el título de concesión del Gobierno del Estado de Sonora por $540 \mathrm{Mm}^{3}$ para uso público urbano, cuya baja está en proceso, e incluyéramos $325 \mathrm{Mm}^{3}$ del DDR 051, que no se encontró entre los datos obtenidos del REPDA, los resultados se modificarían notablemente:

- El volumen de agua para uso público urbano en la cuenca baja no sería de $607.73 \mathrm{Mm}^{3}$ como se indica en Tabla 4, sino de $67.73 \mathrm{Mm}^{3}$. Habría que considerar además los cambios más recientes en las concesiones para abastecer a la ciudad de Hermosillo mediante el acueducto Independencia en los años 2010 y 2011. 
- El volumen de agua para uso agrícola en la cuenca baja sería de $457.8 \mathrm{Mm}^{3}$, y no de $132.8 \mathrm{Mm}^{3}$, como se obtuvo en la tabla.

- Considerando que el total concesionado en la cuenca del río Sonora sería de aproximadamente $812 \mathrm{Mm}^{3}$, el uso agrícola concentraría entonces el $80 \%$ del volumen concesionado, mientras que el uso público urbano tendría el 9\%. Estas proporciones son similares a las reportadas por la Comisión Estatal del Agua en las estadísticas del agua para Sonora en 2008 (CEA Sonora, 2008).

Entonces, la pregunta relevante es si estos volúmenes son sustentables o si se está extrayendo más de la recarga. Para acercarnos a la respuesta recurrimos a los estudios de disponibilidad de agua superficial y de agua subterránea para esta cuenca.

\section{Disponibilidad de agua superficial y subterránea}

Con el fin de tener puntos de comparación que permitan ponderar la pertinencia de los volúmenes de agua concesionados en la cuenca del río Sonora, analizamos la información oficial publicada por la CONAGUA en relación con la disponibilidad de agua en esta cuenca. De acuerdo con los estudios y decretos de disponibilidad de aguas superficiales más recientes para la cuenca del río Sonora, no existe agua superficial disponible en la cuenca en ninguno de sus tramos y esta se encuentra totalmente en déficit, lo cual significa, cuando menos, que se está utilizando toda el agua superficial disponible y que probablemente se usa más de la que está asignada. 
Derechos de agua y gestión por cuencas en México. El caso del río Sonora

Tabla 5. Disponibilidad de aguas superficiales

en la cuenca del río Sonora

\begin{tabular}{|c|c|c|c|c|}
\hline $\begin{array}{l}\text { Nombre } \\
\text { del tramo }\end{array}$ & Segmento de la cuenca & $\begin{array}{l}\text { Superficie de } \\
\text { aportación } \\
\left(K m^{2}\right)\end{array}$ & $\begin{array}{l}\text { Volumen } \\
\text { disponible }\end{array}$ & Situación \\
\hline $\begin{array}{l}\text { Río } \\
\text { Sonora } 1\end{array}$ & $\begin{array}{l}\text { Desde el nacimiento del } \\
\text { río hasta la estación } \\
\text { hidrométrica El Orégano } \\
\text { II }\end{array}$ & 11491.0 & 0.00 & Déficit \\
\hline $\begin{array}{l}\text { Río San } \\
\text { Miguel }\end{array}$ & $\begin{array}{l}\text { Desde el nacimiento del } \\
\text { río hasta la estación } \\
\text { hidrométrica El Cajón }\end{array}$ & 3798.8 & 0.00 & Déficit \\
\hline $\begin{array}{l}\text { Río } \\
\text { Sonora } 2\end{array}$ & $\begin{array}{l}\text { Desde las estaciones } \\
\text { hidrométricas El Orégano } \\
\text { II y El Cajón hasta } \\
\text { la presa Abelardo L. } \\
\text { Rodríguez }\end{array}$ & 5907.3 & 0.00 & Déficit \\
\hline $\begin{array}{l}\text { Río } \\
\text { Sonora } 3\end{array}$ & $\begin{array}{l}\text { Desde la presa Abelardo } \\
\text { L. Rodríguez hasta su } \\
\text { desembocadura en el mar } \\
\text { de Cortés }\end{array}$ & 17576.8 & 0.00 & Déficit \\
\hline
\end{tabular}

Fuente: Comisión Nacional del Agua (2007), DOF, 24 de septiembre de 2007.

Por otra parte, en lo que respecta a las aguas subterráneas, únicamente hay disponibilidad de agua en la cuenca alta, en los acuíferos de los ríos Bacoachi y Bacanuchi, así como en el acuífero de la Poza, mientras que el acuífero de Santa Rosalía está en equilibrio. Sin embargo, los acuíferos de San Miguel, Zanjón, Mesa del Seri-La Victoria y Costa de Hermosillo no tienen disponibilidad y se encuentran en déficit; además, el que se encuentra en el estado más crítico es el de la Costa de Hermosillo, del que se extrae 70\% más que la recarga. 
Tabla 6. Disponibilidad de aguas subterráneas $\left(\mathrm{Mm}^{3}\right)$

\begin{tabular}{|c|c|c|c|c|}
\hline 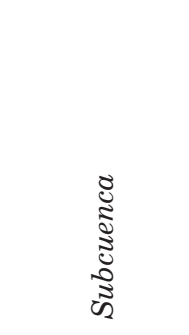 & 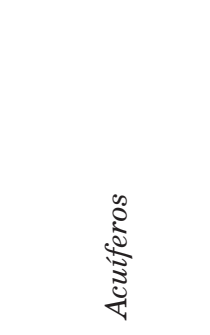 & 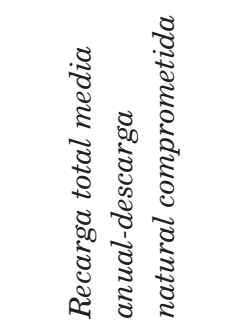 & 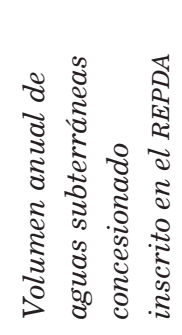 & 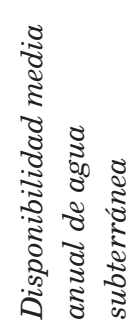 \\
\hline Cuenca alta & $\begin{array}{l}\text { Bacoachi + } \\
\text { Bacanuchi }\end{array}$ & $50.7-6.1=44.6$ & 16.4 & 20.52 \\
\hline $\begin{array}{l}\text { Cuenca } \\
\text { Media }\end{array}$ & Santa Rosalía & 11.8 & 7.4 & 0 \\
\hline San Miguel & San Miguel & 52.5 & 54.0 & -1.5 \\
\hline Zanjón & Zanjón & 76.8 & 90.0 & -13.2 \\
\hline Cuenca Baja & La Poza & $33.8-10=23.8$ & 11.2 & 12.63 \\
\hline \multirow[t]{2}{*}{$\begin{array}{l}\text { Hermosillo- } \\
\text { Costa }\end{array}$} & $\begin{array}{l}\text { Mesa del } \\
\text { Seri-La } \\
\text { Victoria }\end{array}$ & $73-16=57$ & 120.0 & -41.54 \\
\hline & $\begin{array}{l}\text { Costa de } \\
\text { Hermosillo }\end{array}$ & 250.0 & 430.9 & -180.96 \\
\hline
\end{tabular}

Fuente: Comisión Nacional del Agua (2009a y 2009b).

La sobreextracción ocurre principalmente en la cuenca baja, en donde se encuentra la Costa de Hermosillo, que tiene una concesión de $325 \mathrm{Mm}^{3}$, pero que, según Olavarrieta (2010), en 2007 se llegaron a extraer hasta $377 \mathrm{Mm}^{3}$. Más aún, se estima que la recarga del acuífero de esta zona es de $250 \mathrm{Mm}^{3}$, de los cuales alrededor de $100 \mathrm{Mm}^{3}$ provienen de agua del mar (Monreal et ál., 2002). Así, no solamente se extrae más agua de la concesionada, sino que se ha concesionado una cantidad superior a la recarga, generando una explotación no sustentable del acuífero.

Según se observa en estos decretos de disponibilidad, las aguas superficiales están enteramente asignadas. De la misma manera, las aguas subterráneas de los ríos San 
Miguel y Zanjón, así como del acuífero de la Costa de Hermosillo, muestran también elevados déficits con respecto a la recarga. Puede afirmarse, entonces, que las asignaciones de derechos están excedidas, y que también lo están los usos y aprovechamientos del recurso en toda la cuenca. Cada usuario extrae la máxima cantidad de agua para atender sus intereses personales, sin reparar en el equilibrio y conservación de la cuenca. Con este comportamiento es previsible el deterioro general de la cuenca y el agotamiento de los acuíferos, es decir, la tragedia de los comunes.

\section{Conclusiones}

Un problema de política pública bastante conocido y estudiado es el de los recursos de uso común, los cuales, en vista de su carácter colectivo y aparentemente renovable, tienden a ser sobreexplotados y pueden terminar en lo que se conoce como la tragedia de los comunes o, dicho de otro modo, la ruptura del equilibrio natural al deteriorar irreversiblemente el recurso o al agotarlo por completo (Ostrom, 1990).

Entre las opciones de solución para este problema está la intervención estatal. En esa opción el gobierno interviene para poner límites a las extracciones individuales y lograr así un equilibrio colectivo. Este es el tipo de solución que se ha tratado de implantar en México, en donde a nivel legislativo se ha establecido la norma de gestión por cuenca y se ha declarado como de interés público "la protección, mejoramiento, conservación y restauración de cuencas hidrológicas, acuíferos, cauces, vasos y demás depósitos de agua de propiedad nacional, zonas de captación de fuentes de abastecimiento, zonas federales, así como la infiltración natural o artificial de aguas para reabastecer mantos acuíferos" (LAN 2004, art. 7, fracc. II). El control de las concesiones y asignaciones de agua es, por su propia naturaleza, 
el instrumento idóneo para llevar a cabo la gestión por cuencas y tratar de alcanzar los objetivos de interés público de conservación y restauración de los recursos naturales de uso común. Por ello, desde 1994 la Ley de Aguas Nacionales estableció el registro de los derechos de agua.

Sin embargo, en la práctica, llevar a cabo la gestión por cuenca o avanzar hacia la conservación del recurso no es el objetivo del otorgamiento y registro de los títulos de derechos de agua; de acuerdo con lo publicado en documentos oficiales de menor jerarquía jurídica (como la memoria documental y la página web del REPDA), el objetivo de los derechos de agua es dar certeza jurídica de los concesionarios a otros concesionarios. Por ello, el incentivo de los usuarios al registrar sus aprovechamientos y extracciones es contar con dicha seguridad frente a otros usuarios o demandantes de agua y frente a las mismas autoridades. De ese modo, los derechos de agua no se están utilizando como un instrumento para limitar y moderar las extracciones de agua y alcanzar una gestión más sustentable del recurso.

La supuesta concesión (acto de una autoridad que da permiso) es, en realidad, un mero registro, reconocimiento o legalización de usos que ya se están ejecutando. El proceso de registro de concesiones de derechos de agua iniciado en 1994 ha sido solamente un proceso de incorporación de la información de las extracciones que se realizan en la práctica y que ya se venían realizando desde tiempo atrás. Por consiguiente, no se trata de una planeación propiamente dicha, tampoco de decisiones que se toman con base en la disponibilidad; son unas que obedecen más bien a un intento de las autoridades de la CONAGUA por hacer que los usuarios registren sus extracciones y así poder contar con información aproximada de los aprovechamientos y extracciones.

Hay una tensión inherente entre los intereses de los usuarios y las funciones de registro que trata de realizar la CONAGUA. En la práctica la Comisión está subordinada 
a la voluntad de los usuarios para realizar los registros. No hay acciones que denoten una autoridad vertical, por lo que la autoridad no controla el recurso ni decide a quién otorgar los derechos de agua; únicamente toma nota de lo ya existente y consagrado por el derecho consuetudinario.

Por otro lado, los registros de derechos de agua no están agrupados por cuencas hidrológicas, como sería lo esperado de acuerdo con la política de gestión por cuencas, sino por "regiones hidrológicas" que contienen varias cuencas y divisiones políticas (estado o municipio) cuyas demarcaciones no coinciden con las cuencas. Es así que la manera como se lleva a cabo el registro no permite ni facilita la realización de balances de los usos y extracciones del recurso por cuenca. Por ello, a fin de avanzar en la gestión por cuenca, además de la referencia a la división política, deberá hacerse la vinculación o definición de los derechos a la cuenca hidrológica a la que pertenece.

Como se pudo observar, el ejercicio de balance de los derechos de agua en la cuenca del río Sonora, debido a que no está disponible con base en la cuenca, se realizó con base en la división municipal y solo sirve para hacer una aproximación a la contabilidad de los derechos por cuenca. Dicho ejercicio mostró que la mayoría de los registros de derechos de agua se realizaron en la década de los noventa y que para la década de los dos mil los registros se habían reducido considerablemente. Se pudo observar que el 76\% de los volúmenes concesionados corresponden a la cuenca baja, es decir, al municipio de Hermosillo. También se observó que el 59\% del volumen concesionado en la cuenca corresponde a aguas superficiales y que la contraparte, el $41 \%$, corresponde a aguas subterráneas.

Del total del volumen concesionado en los quince municipios estudiados, el 31\% del agua se usa para la agricultura y el $1 \%$ se destina al uso pecuario; el uso en actividades agropecuarias ronda el $32 \%$. Por otra parte, el 60\% está 
destinado al uso público urbano y el 3\% a uso industrial. El restante 5\% puede ser tipificado como de usos múltiples. Con todo, conviene destacar que los datos deben tomarse con precaución, ya que se detectaron ausencias y errores en la base del REPDA. Considerando esto, debe enfatizarse la necesidad de generar información confiable de manera sistemática; una que se actualice con el dinamismo con el que ocurren los cambios en la realidad, pues de otra manera, tanto la investigación académica como la planeación de políticas públicas en materia de gestión del agua consistirán en aproximaciones imprecisas.

La asignación o contabilidad de los derechos puede ser contrastada con las declaraciones oficiales de no disponibilidad y de déficit, tanto de aguas superficiales como subterráneas en los ríos San Miguel y Zanjón, así como en la parte baja de la cuenca. Destaca principalmente el alto déficit del acuífero de la Costa de Hermosillo, cuyo nivel de sobreextración es de más del 70\%. Con esto, se aprecia que los derechos de agua no están sirviendo para limitar las extracciones, puesto que se han otorgado más derechos de agua de los que la conservación y sustentabilidad del recurso aconsejaría; de hecho, lo que hacen estos derechos es dar protección jurídica a las sobreextracciones.

De esta manera, aunque el propósito de velar por el interés común, manifiesto en el artículo 27 constitucional, pudiera interpretarse como una función orientada a la planeación y gestión tendiente a la conservación del recurso mediante la limitación de los excesos del interés individual y la precaución de lo que se ha definido como la tragedia de los comunes, en la práctica tal cautela no existe; al menos no en el caso del río Sonora, donde la intervención estatal no ha servido, hasta ahora, para evitar o impedir el agotamiento del acuífero y la sobreexplotación de la cuenca en general.

Fecha de recepción: 25 de junio de 2013

Fecha de rceptación:11 de julio de 2014 
Derechos de agua y gestión por cuencas en México. El caso del río Sonora

Barkin, D. y T. King, 1975, Desarrollo económico regional: enfoque por cuencas hidrológicas de México, Siglo XXI, México.

Comisión Estatal del Agua y Torynor, 2009, Actualización de estudios básicos y factibilidad para el proyecto ejecutivo de la presa de Sinoquipe, CEA, Hermosillo.

Comisión Estatal del Agua, 2008, Estadísticas del Agua en el Estado de Sonora, CEA, Hermosillo.

Comisión Nacional del Agua, 2007, "ACUERDO por el que se da a conocer el resultado de los estudios de disponibilidad media anual de las aguas", Diario Oficial de la Federación, 24 de septiembre.

__, 2009a, Actualización de la disponibilidad media anual de agua subterránea acuífero (26/9) Costa de Hermosillo estado de Sonora. CONAGUA, México.

__, 2009b, Determinación de la disponibilidad de agua en el acuífero La Poza (2622) estado de Sonora, Gerencia de Aguas Subterráneas, México.

— , s.f., Memoria Documental: Registro Público de Derechos de Agua, Semarnat, México.

Comisión Nacional del Agua y Organismo de Cuenca Noroeste, 2007, Plan director para la modernización integral del riego del Distrito de Riego 05 I - Costa de Hermosillo, Sonora, CONAGUA, OCNO y Colegio de Postgraduados, México.

Diario Oficial de la Federación, 20 I2, "Ley de Aguas Nacionales. Última reforma (08 de junio)”, en línea: http://www. diputados.gob.mx/LeyesBiblio/pdf//6.pdf

__, 20II, "Reglamento de la Ley de Aguas Nacionales. Última reforma (24 de mayo)”, en línea: http://www. diputados.gob.mx/LeyesBiblio/regley/Reg_lan.pdf

— , 2009, "ACUERDO por el que se da a conocer la ubicación geográfica de 37I acuíferos del territorio nacional, se actualiza la disponibilidad media anual de agua subterránea de 282 acuíferos, y se modifica, para su mejor precisión, la descripción geográfica de 202 
Nicolás Pineda, José Luis Moreno, Alejandro Salazar y América Lutz Ley

Bibliografía

acuíferos (28 de agosto)”, en línea: http://dof.gob.mx/ nota_detalle.php?codigo $=5 \mathrm{I}$ 07344\&fecha $=28 / 08 / 2009$

- 2007, "ACUERDO por el que se da a conocer el resultado de los estudios de disponibilidad media anual de las aguas superficiales en las cuencas hidrológicas de los ríos Sonora I, San Miguel, Sonora 2, Sonora 3, Mátape I, Mátape 2, Bavispe, Yaqui I, Yaqui 2, Yaqui 3, de los arroyos Cocoraque I, Cocoraque 2, Río Mayo I, Arroyo Quiriego, Río Mayo 2 y Río Mayo 3, mismos que forman la región hidrológica número 9 denominada Sonora Sur (24 de septiembre)”, en línea: http://dof.gob.mx/nota_detalle.php?codigo $=500$ |436\& fecha $=24 / 09 / 2007$

__, 2006, "Reglamento Interior de la Comisión Nacional del Agua (30 de noviembre)”, en línea: http://www. conagua.gob.mx/CONAGUA07/Noticias/ReglamentoInteriorCONAGUA.pdf

Dourjeanni, A., 2004, Manejo Integral de Aguas en Cuencas: Una forma de gobernabilidad, Comisión Económica para América Latina, Santiago de Chile.

Global Water Partnership, 2009, Handbook for Integrated Water Resources Management in Basins, Scriptoria, Elanders, Sweden.

Hardin, G., 1968, "The Tragedy of the Commons”, Science, núm. 162, Pp. I243-8.

Monreal, R., M. Rangel, J. Castillo, M. Morales, 2002, Estudio de la cuantificación de la recarga del acuífero de la Costa de Hermosillo, municipio de Hermosillo, Sonora, México, Universidad de Sonora, Hermosillo.

Morehouse, Barbara J., Daniel B. Ferguson, Gigi Owen, Anne Browning-Aiken, Pablo Wong-Gonzalez, Nicolás Pineda, Robert Varady, 2008, "Science and socio-ecological resilience: examples from the Arizona-Sonora Border”, Environmental Science \& Policy, núm. II, Pp. 272-284. 